\title{
Improving Instuctions to Stop the Bleed
}

Katherine Forsyth, Bethany Lowndes, Erik Prytz, Carl-Oscar Jonson, Sztajnkrycer Matthew, Stephanie Heller, Susan Hallbeck and Renaldo Blocker

\section{Book Chapter}

Pre-Print

N.B.: When citing this work, cite the original article.

Part of: Proceedings of the Human Factors and Ergonomics Society 2017 Annual Meting. pp. 588-592, 2017,

DOI: https://doi.org/10.1177/1541931213601631

Copyright: Authors and Human Factors and Ergonomics Society, HFES

Available at: Linköping University Institutional Repository (DiVA)

http://urn.kb.se/resolve?urn=urn:nbn:se:liu:diva-141983 


\title{
IMPROVING INSTRUCTIONS TO STOP THE BLEED
}

\author{
Katherine L. Forsyth, $\mathrm{PhD}^{1,2}$, Bethany R. Lowndes, $\mathrm{PhD}^{1,2}$, Erik Prytz, $\mathrm{PhD}^{3,4}$, Carl-Oscar Jonson, $\mathrm{PhD}^{4}$, \\ Matthew D. Sztajnkrycer, MD, $\mathrm{PhD}^{6}$, Stephanie F. Heller, $\mathrm{MD}^{5}$, M. Susan Hallbeck, $\mathrm{PhD}^{1,2}$, \\ Renaldo C. Blocker, $\mathrm{PhD}^{1,2}$
}

\begin{abstract}
${ }^{1}$ Mayo Clinic, Robert D. and Patricia E. Kerns Center for the Science of Health Care Delivery, Rochester, MN; ${ }^{2}$ Mayo Clinic, Department of Health Sciences Research, Rochester, MN; ${ }^{3}$ Linköping University, Department of Computer and Information Science, Linköping, Sweden; ${ }^{4}$ Linköping University, Center for Disaster Medicine and Traumatology, Department of Clinical and Experimental Medicine, Linköping, Sweden; ${ }^{5}$ Mayo Clinic, Department of Emergency Medicine, Rochester, MN; ${ }^{6}$ Mayo Clinic, Department of Surgery, Rochester, MN
\end{abstract}

The Stop The Bleed initiative was developed by the Department of Homeland Security to educate lay providers in bleeding reduction strategies. The current study evaluated: (1)three tourniquet instructions using a simulated tourniquet task and (2)participant confidence levels in tourniquet use and lay provider training. Thirty participants with limited clinical experience applied a tourniquet to a simulated limb using one of three instruction sets. Twelve of these participants (40\%) participated in a tourniquet training session and focus group to discuss each instruction set. Participants preferred the most simple and pictoral instruction set, and identified opportunities for improvement in each set. Participant confidence in tourniquet use increased significantly following the task and the focus group. After the focus group, participant confidence in instructing lay providers on proper tourniquet use significantly increased. Adding key steps, contextual pictures, and indicators of success to instructions could support lay providers stop the bleed in life-threatening situations.

\section{INTRODUCTION}

Following an increase in mass casualty events in the United States, national directives called for public preparedness in simple techniques to stop life-threatening bleeding (American College of Surgeons, 2016; Frangou, 2016; U.S. Department of Homeland Security, 2015). This includes tasks such as applying continuous pressure to a wound, packing a wound with gauze, and applying a tourniquet (Pons \& Jacobs, 2016). A national survey indicated the public is willing to help stop life-threatening bleeding (Jacobs, Burns, Langer, \& Kiewiet de Jonge, 2016); however, the education opportunities are limited. Therefore, organizations such as the American College of Surgeons (ACS) and Hartford Consensus have responded to the call by urging surgeons to train their communities on bleeding reduction strategies, especially tourniquet use (Frangou, 2016).

Tourniquet use has historically been a controversial issue. Doctors were traditionally taught to avoid tourniquet use for fear of nerve, muscle, and vascular injuries and limb amputation (Lee, Porter, \& Hodgetts, 2007). The American Red Cross First Aid handbook (Handal, 1992) avoided discussing tourniquets, while the seventh edition of the Advanced Trauma Life Support (ATLS) course (American College of Surgeons Committee on Trauma, 2004) explicitly instructed against the use of them (Lee et al., 2007). Yet, the military commonly uses tourniquets for combat trauma and has documented its success (Brodie et al., 2007; Kragh et al., 2011). For example, more recent editions of the ATLS course, published by the ACS, include tourniquet training.

With the DHS "Stop The Bleed" initiative, tourniquets have become an acceptable method to mitigate hemorrhage in the civilian sector, and multiple instructional methodologies have been developed to instruct lay providers in applying a tourniquet. Commercially-available tourniquets come with instruction sets, but both the DHS and the ACS have createdjust-in-time training posters to instruct lay providers (both victims and helpers) on proper tourniquet use. Given that the public is interested in having public access bleeding control kits analogous to automated external defibrillators (AEDs) (Jacobs et al., 2016), and the complex technical nature of current commercial instructions, it is critical to evaluate lay provider instructions in order to ensure the ability for safe and proper tourniquet application.

Based upon their experience in the management of trauma, the ACS expects surgeons to train or be able to train lay providers in tourniquet use and other bleeding reduction strategies. However, civilian surgeons themselves may lack the knowledge and training necessary to educate communities on tourniquet use. Using instructions developed for ACS and DHS initiatives and those provided with a tourniquet, this study sought to evaluate: (1) currently available tourniquet instructions through a tourniquet task; and (2) participant confidence levels in tourniquet use and tourniquet training with a group of future surgeons.

\section{METHODS}

This prospective, comparative study was conducted with general surgery residents (postgraduate [PGY] 1), medical students, and research trainees in the simulation center of an academic, tertiary care center. In the first part of this study, participants performed a tourniquet task using one of three instruction sets. In the second part of the study, participants received tourniquet education and participated in a focus group to identify opportunities for instruction improvement. Participants were allowed to opt out at any time.

Tourniquet performance task research protocol. The tourniquet task was one component of an assessment on clinical knowledge, decision-making, and technical skills held over an eight-hour period. Participants understood their 
participation and performance in the study did not contribute to the larger clinical skills test.

At the beginning of the clinical assessment day, participants completed a survey that collected demographics, and previous experience with tourniquets. An additional question included confidence in tourniquet application, answered on a 5-point Likert scale ( $1=$ Not at all confident, $5=$ Extremely confident). The study room was partitioned so that each participant was unable to see the performance task when entering the room. At this time, the participant received the introduction and task scenario. When participants went beyond the partition, they were presented with a lower body simulator with an open wound in one leg, a Combat Application Tourniquet Generation 7 (CAT-7) tourniquet, a marker, and one set of instructions (Figure 1). In the scenario presented to the participant, the victim demonstrated exsanguinating extremity hemorrhage (i.e., bleeding out) and tourniquet application was required to "stop the bleed." Participants had three minutes to read the instructions and apply the tourniquet correctly. At the end of three minutes, participants rated their confidence and satisfaction in applying a tourniquet, as well as their satisfaction in the instructions that were provided using a 5point Likert scale $(1=$ Not at all confident, $5=$ Extremely confident; $1=$ Not at all satisfied, 5=Extremely satisfied). The last question sought feedback on how to improve the provided instructions through more or less words, pictures, or steps. An 'other' option was also available for open response.

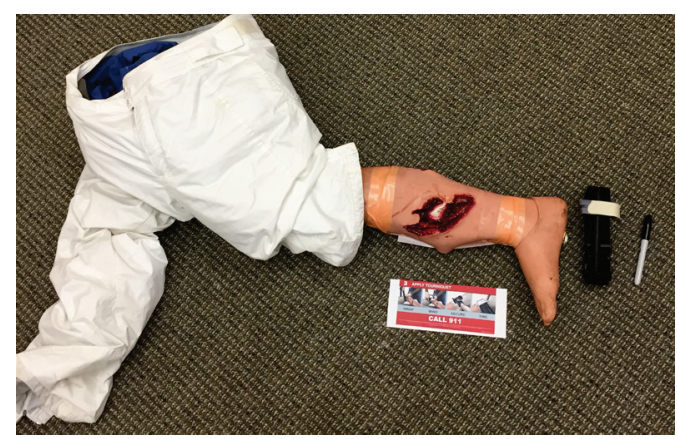

Figure 1. Simulated scenario presented to participants.

Instructions. Instructions developed by the ACS, DHS, and the CAT-7 manufacturer were used in this study. The instructions developed by ACS and DHS included additional procedure steps; however, steps not pertaining to tourniquet placement (e.g., applying pressure) were removed to ensure participants only received information on tourniquet application for each source. Participants were randomized to different instructions according to gender and position (resident, fellow, or medical student) to achieve a near balanced sample size for each group.
A

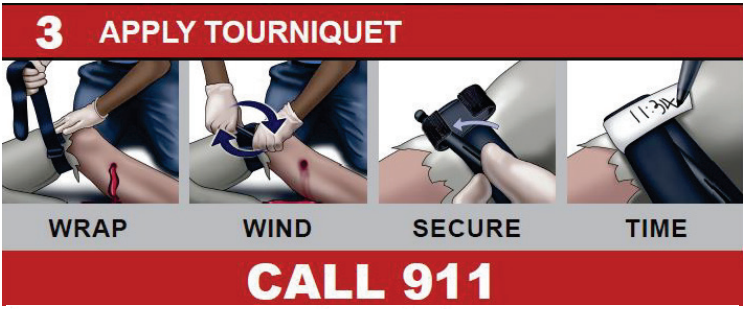

B

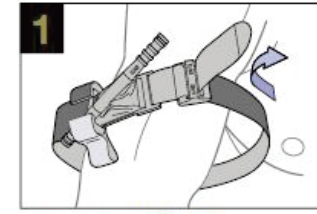

TWO-HANDED APPUCATION Route the band around the limb, pass the tip through the slit of the buckle, and position the CNT $2 \cdot 3^{\prime \prime}$ above the bleeding site directly to the skin.*
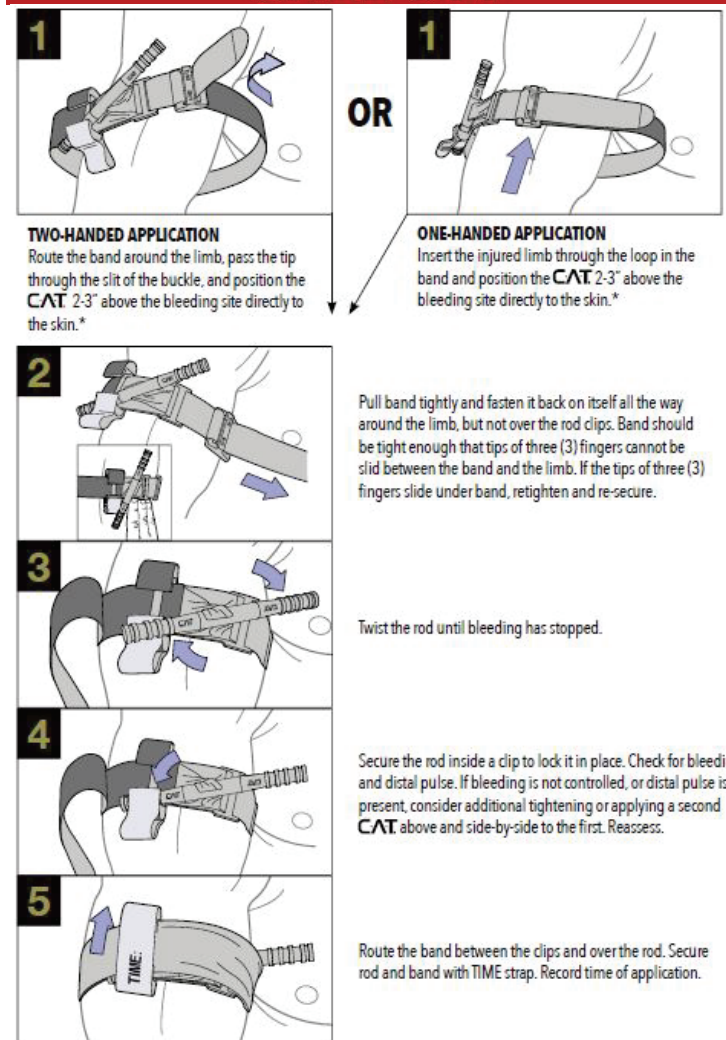

Pull band tightly and fasten it back on itself all the way around the limb, but not over the rod dips. Band should be tight enough that tips of three (3) fingers cannot be
slid between the band and the limb. If the tips of three (3) fingers slide under band, retighten and re-secure.

Twist the rod until bleeding has stopped.

Secure the rod inside a clip to lock it in place. Check for bleeding and distal pulse. If bleeding is not controlled, or distal pulse is present, consider additional tightening or applying a second

Route the band between the clips and over the rod. Secure rod and band with TME strap. Record time of application.

\section{3. Apply Tourniquet(s)}

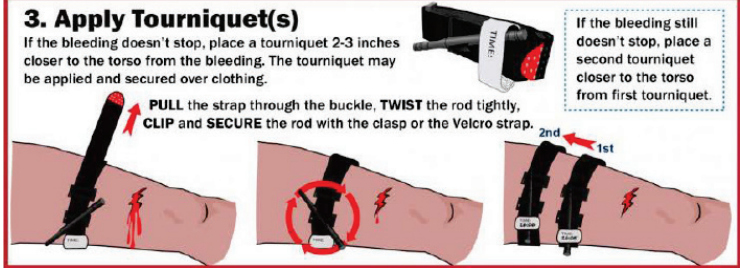

Figure 2. Tourniquet instructions developed by the American College of Surgeons (A), the Combat Application Tourniquet (B), and the Department of Homeland Security.

Training and focus group research protocol. One month following the task, convenience sampling was used to give participants another opportunity to learn about tourniquet use and provide feedback on the instructions. At the beginning of this session, participants answered two survey items on confidence in applying a tourniquet and confidence in teaching tourniquet use using a 5-point Likert scale $(1=$ Not at all confident, 5=Extremely confident). Participants then received 30-minutes of training from an experienced, trauma and general surgery physician (SFH) through video instruction 
and didactics on correct application steps and indicators of success.

Following training, two researchers (KLF, BRL) led a focus group for 30 minutes that reviewed the instructions used in the original tourniquet performance task. The intent of the focus group was to clarify responses from the post-tourniquet-taskperformance survey and to allow individual participants to compare all three instruction sets as each only viewed one instruction set during the performance task. Participants were presented each instruction set and were asked what aspects were successful, unclear, or could use improvement. Participant comments were logged by additional researchers (NES, RCB, MSH). After the focus group, participants again answered two survey items on confidence in applying a tourniquet and confidence in teaching tourniquet use using a 5 -point Likert scale $(1=$ Not at all confident, 5=Extremely confident).

Data analysis. Demographic data was evaluated using descriptive statistics. Paired t-tests evaluated participant responses to survey questions pre- and post-task and pre- and post-focus group. A one-way analysis of variance (ANOVA) was conducted to determine instruction satisfaction for each instruction group. Spearman's correlations were used to test associations between pre- and post-focus group confidence in using and teaching on tourniquets.

\section{RESULTS}

Demographics. Residents $(n=26)$, medical students $(n=1)$, and research trainees $(n=3)$ participated in the tourniquet performance task. Seventeen participants (56.7\%) reported no previous experience applying tourniquets. In contrast, one participant reported previous experience included five tourniquet applications. Those who had previous training on tourniquets $(n=13,43.3 \%)$ most commonly received the training through the ACS-Advanced Trauma Life Support (ATLS) course. Participants who had previously received tourniquet training rated themselves as more familiar with applying a tourniquet $(\mathrm{M}=3.00, \mathrm{SD}=0.68)$ than those who had not previously received training $(\mathrm{M}=1.94, \mathrm{SD}=0.99), \mathrm{t}(28)=$ $3.357, p<0.01$. They also rated themselves more confident $(\mathrm{M}=2.50, \mathrm{SD}=0.94)$ compared to those who had no training $(\mathrm{M}=1.76, \mathrm{SD}=0.97), \mathrm{t}(29)=2.129, p=0.042$.

Confidence change. Participants were generally more confident in applying a tourniquet after the performance task $(\mathrm{M}=3.17, \mathrm{SD}=1.15)$ as opposed to before $(\mathrm{M}=2.10, \mathrm{SD}=1.03)$, a statistically significant increase of 1.07 (95\% CI, .618 to 1.515 ) points, $t(29)=4.862, p<0.01, d=0.89$. However, only participants with the ACS $(\mathrm{M}=1.56, \mathrm{SD}=1.33)$ or DHS $(\mathrm{M}=1.10, \mathrm{SD}=1.10)$ instructions experienced significant improvement in their confidence post-task on the day of the simulation $(\mathrm{t}(8)=3.5, \mathrm{p}<.01 ; \mathrm{t}(9)=3.16, \mathrm{p}=0.012$, respectively).
Instructions and performance satisfaction. Most participants were slightly $(n=8)$, somewhat $(n=9)$, or moderately $(n=7)$ satisfied in their tourniquet application after the performance task (Figure 3). However, there were no significant differences in instruction satisfaction or satisfaction of tourniquet application across the three instruction types or training levels (i.e. trained, untrained).

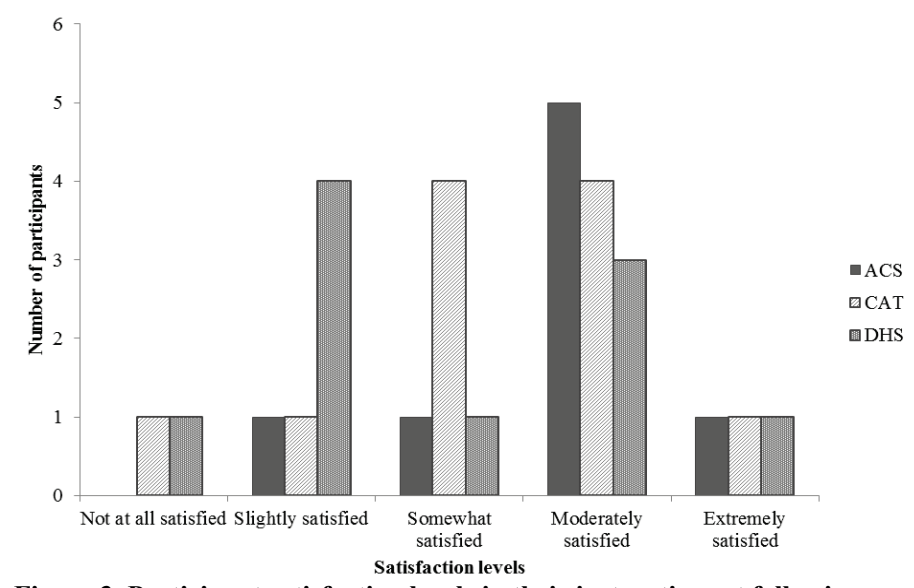

Figure 3. Participant satisfaction levels in their instruction set following the tourniquet performance task.

Improvements to instructions. Only four participants overall reported no desired changes to the instructions. Participants generally reported wanting more pictures, steps, and words overall (68.4\% instances). The most common request for improvement of the ACS instructions was for more pictures (40\%). Participants requested more pictures (28.6\%) but fewer words $(28.6 \%)$ when assessing the CAT-7 instructions, while both more pictures $(42.9 \%)$ and more words $(21.4 \%)$ were requested for the DHS instruction set. In free response, participants also recommended having access to video instructions while applying a tourniquet, and providing indicators for successful application.

Focus group. Twelve of the participants from the tourniquet performance task received training and participated in the focus group. There was no significant difference in participant post-performance task confidence scores and their pre-training confidence scores in applying a tourniquet $(p>0.05)$. In a unanimous vote, all participants preferred the ACS instructions over the other instruction types. Participants reported the ACS instructions (Figure 2A) were "easy" and "simple"; however, participants reported that the instructions should also include "things to check for" such as what direction (proximal) the tourniquet should be placed. One ACS evaluation commented, "This is good, hits the main points. There are two things missing: 1) missing step between wrap and wind, 2) missing tightness of Velcro. Maybe show in extra picture the two figures with securing the Velcro strap."

Participants reported the CAT-7 instructions (Figure 2B) as "way too much." They vocalized the text was "overwhelming" 
and that the "text was so small I felt I had to pick it up to see it and drop the tourniquet." Participants were also confused by the self-application aspect of the instructions that distinguished between 1-handed and 2-handed instructions. The verbiage could also be improved regarding tourniquet placement 'above the wound.' The focus group noted that users could confuse this as tourniquet placement on top of the wound. Additionally, the pictures in the CAT instructions "[do] not show [the tourniquet] in relation to the wound."

The DHS instructions (Figure 2C) made participants "frustrated." Participants felt there were "a lot of words... [that] don't go along with the pictures" and that the "perceptual flow is lost and unclear." Two issues emerged for the participants with this instruction set. The first issue related to the time strap. There was "no obvious sign that [the time] was supposed to be written down." Additionally, "The time strap is applied on every picture, [so] how do they know when to apply this strap?" The second issue related to applying a second tourniquet. "The third picture made me think that I had to move [the tourniquet]." Participants suggested "removing the arrow from the third picture" and "grey-out the second tourniquet and label if first failed."

Following the training and focus group, participants were more confident in applying a tourniquet $(\mathrm{M}=4.41, \mathrm{SD}=.90)$ and teaching tourniquet application to community members $(\mathrm{M}=4.41, \mathrm{SD}=.90)$, a statistically significant increase of 1.08 (95\% CI, .580 to 1.587$)(t(11)=4.733, p=.001, d=0.98)$ and 1.42 points $(95 \% \mathrm{CI}, .500$ to 2.334$)(t(11)=3.4, p=.006$, $d=1.37$ ), respectively, compared to pre-training and focus group confidence levels. Comparing across the entire study, the 12 participants that performed the task, received training, and participated in the focus group rated their confidence in applying a tourniquet 2.58 points higher, a significant increase from their pre-task confidence levels $(t(11)=7.685, p<0.005$, $d=0.45$ ). A strong, positive correlation was observed between participant confidence ratings in applying a tourniquet and confidence in teaching tourniquet use to lay providers or community members, $r_{s}(10)=.725, p=.008$.

\section{DISCUSSION}

As aforementioned, the data were collected during a simulated tourniquet performance task and a focus group. Results suggested that the three instruction types evaluated yielded different participant confidence levels in the tourniquet performance task and similar satisfaction levels in the instructions and their tourniquet application. In general, participants preferred the ACS instruction set over the DHS and CAT-7 instructions used in this study, but identified areas of improvement for each set.

Prior to the performance task, approximately $43 \%$ of participants reported previous training in tourniquet use. Trained participants initially had more confidence and familiarity with tourniquet use; however, subsequent training had no effect on perceptions of the three different instruction types. Participants with prior training may not have achieved tourniquet mastery in their reported previous training, but the similarity in satisfaction responses with non-trained participants indicated general opportunities for improvement for each instruction set.

The ACS set provided detailed, colored pictures with minimal verbiage across four steps. In the survey, ACS participants generally wanted more pictures, words, and steps. This was supported in the focus group where participants vocalized including two missing steps and developing an associated list for success indicators. Participant confidence in tourniquet use also increased the most following the use of ACS instructions, which may reinforce the focus group's overwhelming preference for the ACS set.

The CAT-7 instructions paired smaller pictures with sentences across five steps and included instructions for both twohanded or one-handed use of the tourniquet. Reviewing the CAT-7 instructions yielded mixed results. In the survey, CAT7 participants wanted both more and fewer words, but also more steps and pictures. Outcomes from the focus group suggest making the text larger and reducing it, improving the pictures, and re-phrasing certain verbiage to reduce improper tourniquet use. However, it must be noted that the CAT-7 instructions reflected manufacturer medical device instructions for use as part of a comprehensive training curriculum, rather than the just-in-time training reflected in the ACS and DHS instruction sets.

Lastly, the DHS instructions did not outline each step, but instead paired four colored, low-fidelity pictures with several sentences. The survey and confidence data for the DHS instructions most conflicted with focus group data. Participants using the DHS instructions in the tourniquet performance task reported improved confidence; however in the focus group they vocalized their frustration with the instruction set. While two participants did not wish to change the instructions, others wanted more pictures and more words. In the focus group, participants expressed a need for improved structure and modification of the instructions to better describe time notation and application of a second tourniquet.

In the focus group, participants recognized that lay providers would be the primary users of instructions as just-in-time training. They recommended that all instruction sets should emphasize the location of tourniquet placement with respect to the wound. Given that such providers would likely have no medical knowledge, using technical jargon such as "distal" would not necessarily be effective based on the knowledge level of the user. Rather, simple terms such as "arms" and "legs" should be used. Additional improvements included identifying body parts where tourniquets should not be used 
and indicators of successful placement and tourniquet aftercare. While the curriculum of tourniquet training was not the focus of this study, the formal training received prior to the focus group significantly improved confidence in tourniquet application. The results highlight the utility of formal tourniquet training in preparation for mass violence events, analogous to the current emphasis on CPR and AED training for sudden cardiac death. Prior knowledge might mitigate some of the concerns and uncertainties associated with all 3 instruction types.

This study identified valuable improvements to tourniquet instructions, yet some limitations were unavoidable. This study was performed at one institution with participants of similar medically-trained experience, thus limiting its generalizability. Future work should diversify the sample to include both medically and non-medically trained individuals of varying experience levels across multiple locations. Selfreported previous tourniquets experience was used resulting in portential to over- or under-represent actual experience. Grouping participants by any experience (trained) and no experience (untrained) assisted in reducing this issue. Additionally, it was unclear what types of tourniquets participants used previously. Certain comments, including the desire for video instructions, reflect the artificiality of the training venue. Watching a video during an actual mass casualty event is unrealistic; however, using audio prompts would enable the lay provider to focus on the patient rather than on training instructions. Participants involved in the focus group were chosen out of convenience and may not been an appropriate representation of the task participants. However, this was overcome by encouraging participants to vocalize positives and negatives about each instruction set regardless of their exposure to it in the tourniquet task.

\section{CONCLUSION}

While exposure to tourniquets and minimal didactic training improved participants' confidence in applying tourniquets and training on tourniquet use, existing instructions that facilitate tourniquet applications need to be improved. With the initiative for public education on tourniquet use, instructions should support victims or first-responder laypeople under the assumption that no education has been received. Developing tourniquet instructions that emphasize steps with colored pictures, effective words, and include things to check for along the way to ensure successful tourniquet application.

\section{REFERENCES}

American College of Surgeons. (2016). Did You Know? Stop the Bleed and BleedingControl.org. The Cutting Edge: Post-Clinical Congress 2016 Edition. Retrieved from https://www.facs.org/publications/newsletters/the-cuttingedge/postcc2016/dyk-stop-the-bleed

American College of Surgeons Committee on Trauma. (2004). Advanced Trauma Life Support for Doctors--ATLS: Student Course Manual (7th ed.). Chicago, IL: American College of Surgeons.

Brodie, S., Hodgetts, T. J., Ollerton, J., McLeod, J., Lambert, P., \& Mahoney, P. (2007). Tourniquet use in combat trauma: UK military experience. Journal of the Royal Army Medical Corps, 153(4), 310-313.

Frangou, C. (2016). 'Stop the Bleed' Campaign Focuses on Saving Lives With Simple Techniques [Press release]. Retrieved from http://www.generalsurgerynews.com/In-the-News/Article/12-16/Stop-the-Bleed-Campaign-Focuses-on-Saving-Lives-With-SimpleTechniques $/ 38809 / \mathrm{ses}=$ ogst?enl $=$ true

Handal, K. (1992). The American Red Cross first aid \& safety handbook. Boston: Little, Brown and Company.

Jacobs, L. M., Burns, K. J., Langer, G., \& Kiewiet de Jonge, C. (2016). The Hartford Consensus: A National Survey of the Public Regarding Bleeding Control. Journal of the American College of Surgeons, 222(5), 948-955.

doi:http://dx.doi.org/10.1016/j.jamcollsurg.2016.02.013

Kragh, J. F., Littrel, M. L., Jones, J. A., Walters, T. J., Baer, D. G., Wade, C. E., \& Holcomb, J. B. (2011). Battle casualty survival with emergency tourniquet use to stop limb bleeding. The Journal of Emergency Medicine, 41(6), 590-597.

Kragh J.F., Walters T.J., Baer D.G., Fox C.J., Wade C.E., Salinas J. \& Holcomb J.B. Survival with emergency tourniquet use to stop bleeding in major limb trauma. 2009. Ann Surg. 249: 1-7.

Kragh J.F, Walters T.J, Baer D.G, Fox C.J., Wade C.E., Salinas J, \& Holcomb J.B. (2008). Practical use of emergency tourniquets to stop bleeding in major limb trauma.J Trauma, 64: S38-S50.

Lee, C., Porter, K. M., \& Hodgetts, T. J. (2007). Tourniquet use in the civilian prehospital setting. Emergency Medicine Journal, 24, 584-587. doi:10.1136/emj.2007.046359

Pons, P., \& Jacobs, L. (2016). Save a life: What everyone should know to stop bleeding after an injury. A. C. o. Surgeons (Ed.)

U.S. Department of Homeland Security. (2015). Presidential Policy Directive / PPD-8: National Preparedness. Retrieved from https://www.dhs.gov/presidential-policy-directive-8-nationalpreparedness 